Cahiers de philosophie de l'université de

\title{
Avant-propos : La totalité est-elle un tout comme un autre?
}

Maud Pouradier

\section{(2) OpenEdition}

1 Journals

Édition électronique

URL : https://journals.openedition.org/cpuc/335

DOI : $10.4000 /$ cpuc.335

ISSN : 2677-6529

Éditeur

Presses universitaires de Caen

\section{Édition imprimée}

Date de publication : 31 décembre 2016

Pagination : 7-12

ISBN : 978-2-84133-842-9

ISSN : 1282-6545

\section{Référence électronique}

Maud Pouradier, « Avant-propos : La totalité est-elle un tout comme un autre? ?, Cahiers de philosophie de l'université de Caen [En ligne], 53 | 2016, mis en ligne le 01 février 2019, consulté le 31 janvier 2023. URL : http://journals.openedition.org/cpuc/335 ; DOI : https://doi.org/10.4000/cpuc.335

\section{(c) (7) (8)}

Creative Commons - Attribution - Pas d'Utilisation Commerciale 4.0 International - CC BY-NC 4.0 https://creativecommons.org/licenses/by-nc/4.0/ 


\section{Avant-propos: La totalité est-elle un tout comme un autre?}

$\mathrm{D}$ ANS la Dissertation DE 1770, Kant écrit que la «totalité absolue, bien qu'elle ait l'apparence d'un concept courant et facile [...] est une croix pour le philosophe ${ }^{1}$. Alors que l'ensemble total $\left(\right.$ universitas $^{2}$ ) de tout ce qui existe semble saisissable par le concept de tout (totum), sa synthèse effective sous la condition du temps est inaccessible ${ }^{3}$. Déchirée entre simplicité du tout abstrait et complexité infinie de la totalité synthétique, la raison, au croisement de concepts qui ne se recoupent pas, est au supplice. Les antinomies cosmologiques en déploieront les quatre modalités dans la Critique de la raison pure. La totalitas absoluta est une croix, car sa série complète ne peut jamais constituer un totum ${ }^{4}$. Mais le problème ne vient-il pas de la volonté dogmatique d'assimiler des concepts qui ne peuvent que s'entrecroiser? Et faut-il nécessairement que la totalité se réduise au tout, au point de devenir son synonyme?

Assimiler la totalité au tout revient à confondre ce à quoi rien ne manque avec ce qui a toutes ses parties. Dans le Parménide, le jeune Socrate ne discerne pas l'alternative, et dessine ainsi pour longtemps l'histoire du concept de totalité. Dans le dialogue de Platon, le vieux dialecticien commençait en effet par mettre en difficulté le jeune philosophe à propos de la participation:

Est-ce donc au tout de la Forme ou à l'une des parties de cette Forme que participe chaque chose qui participe à cette Forme? Ou y aurait-il une autre façon de participer, à part ces deux-là 5 ?

1. Kant, La Dissertation de 1770, P. Mouy (trad.), Paris, Vrin, 1995, p.31 et 33. Traduction légèrement modifiée. Le terme latin est totalitas absoluta.

2. Ibid., p.30.

3. Ibid., p. 32 .

4. Ibid

5. Platon, Parménide, L. Brisson (trad.), Paris, Flammarion, 1994, 131 a, p. 94-95. Traduction légèrement modifiée. La méréologie du Parménide est reconstituée dans V. Harte, Plato on Parts and Wholes, Oxford - New York, Clarendon Press, 2002. 
Socrate propose alors l'image du jour, qui semble pouvoir défier les relations entre le tout et les parties, puisqu'il reste un en éclairant divers lieux. Et Parménide de modifier l'image du jour en celle du voile, qui se divise en autant de portions que de choses voilées. Si Parménide transforme ainsi l'image socratique, c'est qu'il ne conçoit pas qu'un tout ne soit pas divisible. Un peu plus loin dans le dialogue, lorsque le jeu dialectique des hypothèses aura véritablement commencé, il affirmera au jeune Aristote, qui lui aussi n'opposera aucune résistance:

Mais qu'est-ce que le tout? N'est-ce pas ce à quoi aucune partie ne manque, qui peut être considéré comme un tout? [...] Par voie de conséquence, l'un serait composé de parties ${ }^{6}$.

Mais ce à quoi rien ne manque, qu'Albert le Grand ou Thomas d'Aquin appelleront le parfait, est-il nécessairement composé de parties? Dire négativement qu'un tout n'exige aucune partie supplémentaire, est-ce pour autant dire que n'importe quel tout est composé de parties? Tout le néoplatonisme défendra l'image socratique d'un jour auquel rien ne manque. Cette présence plénière qui ne se partitionne pas prendra le nom de holotes dans le $\$ 69$ des Éléments de théologie de Proclus. Cette acception spécifique est en concurrence avec l'acception aristotélicienne de la totalité en Métaphysique Delta, qui fait de la totalité la simple quiddité du tout.

Le concept néoplatonicien de holotes acquiert une nouvelle signification dans le contexte chrétien, en particulier chez celui que la tradition connaît sous le nom de Denys l'Aréopagite. La foi chrétienne et le dogme trinitaire trouvent dans le néoplatonisme un étrange anologon, ou plutôt un symétrique inversé. Pour reprendre l'expression de Paul Aubin, le néoplatonisme voit plusieurs principes là où il n'y en a qu'un, mais il voit de la simple unité là où il y a en effet Trinitér. Denys, lecteur de Proclus, va donc utiliser les modes de pensée néoplatoniciens tout en les reconfigurant. Un de ces déplacements consiste à secondariser le nom d'Un. Dans cette reconfiguration des thèses néoplatoniciennes apparaît l'idée de totalité: Denys parle de Dieu comme d'une holotes. Pourrait-on croire un seul instant que Denys aurait voulu dire que Dieu est un tout que l'on peut découper en parties, alors qu'il consacre tout le second chapitre des Noms divins à expliquer dans quelle mesure les noms par lesquels on loue Dieu s'appliquent pleinement à chacune des personnes trinitaires? Il est donc manifeste qu'il reprend cette notion à Proclus (dont il est un lecteur attentif)

6. Platon, Parménide, $137 \mathrm{c}-\mathrm{d}, \mathrm{p} .114-115$.

7. P. Aubin, Plotin et le christianisme. Triade plotinienne et Trinité chrétienne, Paris, Beauchesne, 1992. 
en tant qu'elle se distingue du holon. Au fond, que Dieu soit à la fois Un et Totalité (et non pas Un et Tout comme le pan parménidien) signifie qu'il est Trinité, ou plutôt Théarchie dans le vocabulaire de Denys:

[L]es Écritures usent toujours des noms de Dieu qui sont dignes de lui pour célébrer indistinctement la Déité pleine et entière, totale et indivisée, attribuant ces noms totalement, indistinctement, absolument, indifféremment, universellement à l'entière totalité de la Déité totale et entière [hototeti tes holotelous kai pases theothetos]. Ainsi [...] ne point attribuer ce nom à la Déité entière, ce serait blasphémer et déchirer par une audace criminelle l'Unité qui transcende toute unité ${ }^{8}$.

Et à la fin du chapitre II :

[T] out nom correspondant à un don de la Bonté divine, à quelque Personne [hypostasis] théarchique qu'il s'applique, peut s'étendre sans scrupule à la totalité théarchique [thearchichès holotetos] ${ }^{9}$.

Même si holotes ne fait pas partie de la liste des noms divins étudiée par Denys (puisqu'il ne s'agit pas d'un nom biblique), il apparaît clairement que ce terme est préférable à celui de holon pour décrire la Déité. Pour Denys, le propre de la totalité semble de n'être pas un tout ou de dépasser toute logique méréologique.

Au chapitre XI des Noms divins, consacré à la paix, Denys utilise de nouveau le concept de holotes: "Car la totalité de la paix intégrale [pantelous eirenes holotes] va à l'intime de tous les êtres par la présence très simple, incorruptible, de sa force unifiante ${ }^{10}$. Or Jean le Sarrazin, dans sa traduction des Noms divins dans les années 1160, traduit ce passage en inversant délibérément en latin le génitif: "Transit enim perfectae totalitatis pax ad omnia exsistentia ${ }^{11}$, c'est-à-dire «la paix de la totalité parfaite coule vers tous les existants». Le Sarrazin va plus loin que la lettre de Denys, en considérant que holotes est un autre nom de la Théarchie: "totalité de la paix » devient la «paix de la totalité», la paix de ce dont elle procède. Ce déplacement grammatical et conceptuel, opéré par le Sarrazin, est de grande importance, sa traduction étant la principale source d'Albert le Grand et de Thomas d'Aquin concernant les Noms divins.

8. Euvres complètes du Pseudo-Denys l'Aréopagite, M. de Gandillac (trad.), Paris, Aubier, 1943, 636c-637a, p. 77-78.

9. Ibid., $637 \mathrm{c}$, p. 79 .

10. Dionysiaca. Recueil donnant l'ensemble des traductions latines des ouvrages attribués à Denys de l'Aréopage, P. Chevalliers (dir.), t. I, Bruges, Desclée de Brouwer, 1937, p. 505. Traduction légèrement modifiée. Euvres complètes du Pseudo-Denys..., 952a, p. 166.

11. Ibid. 
Telle est l'hypothèse qui présida au colloque des 4 et 5 février 2015, tenu à la Maison de la recherche en sciences humaines de l'université de Caen Normandie: celle d'un concept néoplatonicien, distinct de la problématisation aristotélicienne du tout, refondée dans le cadre d'une réflexion trinitaire, qui aurait connu une résurgence au Moyen Âge, notamment grâce aux traductions de Denys par le Sarrazin et de Proclus par Guillaume de Moerbeke. Résurgence qui connaît pourtant immédiatement une éclipse partielle: tout d'abord du fait de la conceptualisation aristotélicienne de la totalité, fort différente, et qui est également approfondie au XIII ${ }^{\mathrm{e}}$ siècle. Ensuite, du fait de la tradition de réflexion méréologique, dont le De divisione de Boèce propose une présentation systématique, et qui s'articule étroitement à la querelle des universaux, dans le creuset de l'Isagoge de Porphyre traduit en latin par le même Boèce. Cette histoire complexe de l'articulation du problème de l'universel à celui du tout a été parfaitement restituée par Alain de Libera dans La Querelle des universaux ${ }^{12}$ : parce que le rapport de l'universel aux particuliers est identifié à celui d'un tout à ses parties, la question de l'universel ne se détache plus des questions méréologiques. Il semble cependant, et telle est la seconde hypothèse de ce colloque, que le concept de totalité ne peut être réduit à cette querelle des universaux d'une part, ou aux discussions strictement méréologiques d'autre part, et qu'il mérite un traitement particulier. Qu'une étude de ce concept nécessite de se référer à ces interrogations particulières ne signifie pas qu'il s'y réduise. Peut-être même risque-t-on, en procédant ainsi, de laisser échapper l'essentiel de cette notion. Ces hypothèses animèrent le colloque de février 2015, et donnent lieu aujourd'hui à la publication de ce dossier, qui pose quelques jalons de cette histoire conceptuelle de la totalité. Anca Vasiliu, à travers l'étude du Livre des XXIV philosophes (à la datation et à l'origine incertaines), resitue la question de la totalité dans son cadre néoplatonicien, et discute les résonances philosophiques et peut-être chrétiennes du terme de totalitas. Dans une perspective articulant néoplatonisme et sources aristotéliciennes, Avicenne pense la question de la création de la totalité de l'être. On connaît l'importance de l'Avicenna latinus pour le XIII ${ }^{\text {e }}$ siècle: Olga Lizzini déploie les différentes problématisations de la totalité et leur articulation dans le vaste corpus avicennien, en soulignant les déplacements induits par la traduction latine. À travers le traitement franciscain des questions cosmologiques, Laure Solignac montre l'élaboration du concept de totalité dans la Summa Halensis et chez saint Bonaventure. L'idée bonaventurienne d'une totalité qui s'allie à la

12. A. de Libera, La Querelle des universaux. De Platon à la fin du Moyen Âge, $2^{\mathrm{e}}$ éd., Paris, Seuil, 2014, p. 326. 
simplicité est explicitée par Chirine Raveton dans une étude comparative des approches bonaventurienne et thomiste du problème de la simplicité divine. Quant à moi, j'ai montré la manière dont saint Thomas d'Aquin reformule la typologie boécienne du De Divisione en termes de totalitas, afin de résoudre certains problèmes posés par la psychologie péripatéticienne en régime chrétien, aboutissant à une synthèse fragile entre totalité néoplatonicienne et totalité aristotélicienne. Pierre de Jean Olivi critiquera vigoureusement la conception thomiste de la totalité, comme le montre Christian Rode dans le texte qui clôt le dossier. Le hasard prenant parfois les traits de la providence, ce Cahier de philosophie de l'université de Caen accueille pour son cinquante-troisième numéro un varia intitulé «Totalité et démocratie. Claude Lefort avec et après Marx» de Mattia Di Pierro - preuve du caractère toujours vivant de la question de la totalité.

Maud Pouradier

Normandie Univ, UNICAEN, Identité et Subjectivité (EA 2129), 14000 Caen, France 
